\title{
The Analysis of a HBV Model with Vaccination
}

\author{
Xiaoxia Yuan \\ Department of Applied Mathematics \\ North University of China \\ Taiyuan, Shanxi, P. R. China
}

\author{
Yakui Xue * \\ Department of Applied Mathematics \\ North University of China \\ Taiyuan, Shanxi, P. R. China \\ *Corresponding author
}

\begin{abstract}
In this paper, we introduce an improved hepatitis B virus(HBV) model to discuss the impact of vaccination. The basic reproductive number $R_{0}$ determine the extinction and the persistence of virus infection. When $R_{0}$ is less than one,the diseasefree equilibrium is globally-asymptotically stable and the infection becomes extinct eventually; When $R_{0}$ is greater than one,the unique endemic equilibrium exists and endemic equilibrium is locally asymptotically. The results indicate that vaccination plays an importment role in preventing and controlling the spread of $\mathrm{HBV}$.
\end{abstract}

Keywords-hepatitis b virus; basic reproduction number; globally asymptotical stable

\section{INTRODUCTION}

Recently, mathematical models have been used frequently to study the transmission dynamics of HBV in various regions. Long et al. (2008) considered the mathematical model of CTL immune response to HBV infection [1].Eikenberry et al.(2009)analyzed the hepatitis B virus infection in a delay model[2].Li et al.(2011)analyzed the dynamic behaviors of a HBV infection model with logistic hepatocyte growth and discuss the stability[3].These models provided useful information about the impact of various control measures.

\section{MODEL}

A deterministic compartmental model, a system of ordinary differential equations, is proposed to describe the dynamics of HBV transmission. The HBV transmission is complex and the detailed mechanism remains unclear, for the sake of simplicity, we make some assumptions:

(1)We class all the vertical infected infants into the chronic carriers.

(2)We assume that all the newborns are vaccinated at the same efficacy, since many countries have introducer $\mathrm{HBV}$ vaccination into their nation infant immunization program.

(3)The exposed compartment will shift either to the acute infection individuals or to the chronic HBV carriers, according to the medical journal by $\mathrm{Cao}(2010)$ [4]. Attention that in this paper the chronic HBV carriers includes the so-called medically HBV carriers and the chronic infectious individuals.

Note that Medley et al. considered only five groups and did not distinguish the recovered and vaccinated subgroups[5].In fact , the immunity after recovery is lifetime, while that following vaccination might wane after some time.
We divide the host population into six groups: the susceptible individuals $S$; infected but not yet infectious individuals (exposed) $E$; acute infectious individuals $I$; chronic HBV carriers $C$; recovered individuals $R$; vaccinated individuals $V$.Of the six stages, both acute infection and chronic HBV carriers can spread the disease. $\mu(1-\omega)$ newborns successfully immunized move directly to the immune following vaccination, $\mu \omega v \mathrm{C}$ babies have infected due to vertical transmission and access to chronic carrier class, the lest $\mu \omega(1-v \mathrm{C})$ newborns are unimmunized and become susceptible individual, $\mathrm{q}$ and $\omega$ is less than 1 ,all the parameters are nonnegative.

Based on the characteristics of HBV transmission, the relevant differential equations are

$$
\begin{aligned}
& \frac{d S}{d t}=\mu \omega(1-v C)+\varepsilon V-\beta(I+\alpha C) S-\gamma_{2} S-\mu S \\
& \frac{d E}{d t}=\beta(I+\alpha C) S-\sigma E-\delta E-\mu E \\
& \frac{d I}{d t}=\sigma E-\gamma_{1} I-\mu I \\
& \frac{d C}{d t}=\delta E+q \gamma_{1} I-\mu \omega v C-\gamma_{3} C-\mu C \\
& \frac{d R}{d t}=(1-q) \gamma_{1} I+\gamma_{3} C-\mu R \\
& \frac{d V}{d t}=\mu(1-\omega)+\gamma_{2} S-\varepsilon V-\mu V
\end{aligned}
$$

Where, $\mu$ is the death rate(and equally the birth rate), $\omega$ is the proportion of failure immunization, $v$ is the proportion of children born to carrier mothers who have been infected, $\varepsilon$ is the loss of immunity rate, $\beta$ is the transmission coefficient, $\alpha$ is the infectiousness of the chronic carriers relative to acute infections, $\sigma$ is the rate of exposed individuals becoming acute infectious individuals, $\delta$ is the rate of the exposed individuals becoming chronic carriers, $\mathrm{q}$ is the proportion of acute infection develops to chronic carriers , $\gamma_{1}$ is the rate of moving from acute class to chronic or recovery class, $\gamma_{2}$ is the vaccination rate, $\gamma_{3}$ is the rate of moving from chronic carrier to recovery.We assume all the newborns are vaccinated within 24 hours after birth, all the parameters are nonnegative. 
Because $R$ appears only in the fifth equation of the system(1), we can discuss the following reduced system:

$$
\begin{aligned}
& \frac{d S}{d t}=\mu \omega(1-v C)+\varepsilon V-\beta(I+\alpha C) S-\gamma_{2} S-\mu S, \\
& \frac{d E}{d t}=\beta(I+\alpha C) S-\sigma E-\delta E-\mu E, \\
& \frac{d I}{d t}=\sigma E-\gamma_{1} I-\mu I, \\
& \frac{d C}{d t}=\delta E+q \gamma_{1} I-\mu \omega v C-\gamma_{3} C-\mu C, \\
& \frac{d V}{d t}=\mu(1-\omega)+\gamma_{2} S-\varepsilon V-\mu V .
\end{aligned}
$$

For the biological sense,

$$
\Omega=\left\{(S, E, I, C, V) \mid \begin{array}{l}
S, E, I, C, V \geq 0, \\
S+E+I+C+V \leq \omega
\end{array}\right\}
$$

is a positive invariant set of the system(2).

For convenience, let $a_{1}=\gamma_{2}+\mu, a_{2}=\sigma+\delta+\mu$,

$$
a_{3}=\gamma_{1}+\mu, a_{4}=\gamma_{3}+\mu-\mu \omega \nu, a_{5}=\varepsilon+\mu \text {. }
$$

\section{BASIC REPRODUCTION NUMBER AND EQUILIBRIUM}

System (2) have two equilibrias in $\Omega$ : the disease-free equilibria $P^{0}=\left(S^{0}, 0,0,0, V^{0}\right)$, where

$$
S^{0}=\frac{\varepsilon+\mu \omega}{\varepsilon+a_{1}} \quad V^{0}=\frac{a_{1}-\mu \omega}{a_{1}+\varepsilon}
$$

If $R_{0}>1$, the endemic equilibria $P^{*}=\left(S^{*}, E^{*}, I^{*}, C^{*}, V^{*}\right)$

$$
\begin{gathered}
S^{*}=\frac{a_{2} a_{3} a_{4}}{\beta \sigma a_{4}+\beta \alpha \sigma a_{3}+\beta \alpha \sigma q \gamma_{1}}=\frac{S^{0}}{R_{0}} \\
E^{*}=\frac{a_{3} a_{4}}{a_{3} \delta+q \gamma_{1} \sigma} C^{*} \quad I^{*}=\frac{a_{4} \sigma}{a_{3} \delta+q \gamma_{1} \sigma} C^{*} \\
C^{*}=\frac{\left(\varepsilon+a_{1}\right) \mu S^{0}}{a_{5} \mu \omega v\left(a_{3} \delta+q \gamma_{1} \sigma\right)+a_{2} a_{3} a_{4} a_{5}}\left(1-\frac{1}{R_{0}}\right) \\
V^{*}=\frac{\mu(1-\omega)+\gamma_{2} S^{*}}{a_{5}}
\end{gathered}
$$

Using the notation in van den Driessche and Watmough (2002) [6], we obtain the basic reproduction number $R_{0}$

$$
\begin{gathered}
F=\left(\begin{array}{ccc}
0 & \beta S^{0} & \beta \alpha S^{0} \\
0 & 0 & 0 \\
0 & 0 & 0
\end{array}\right), V=\left(\begin{array}{ccc}
a_{2} & 0 & 0 \\
-\sigma & a_{3} & 0 \\
-\delta & -q \gamma_{1} & a_{4}
\end{array}\right) \\
R_{0}=\rho\left(F V^{-1}\right)=\frac{a_{4} \beta \sigma S^{0}+\beta \alpha \sigma q \gamma_{1} S^{0}+a_{3} \beta \alpha \delta S^{0}}{a_{2} a_{3} a_{4}} .
\end{gathered}
$$

\section{STABILITY OF EQUILIBRIUM}

Theorem 4.1 For the system (2.2)

(1) If $R_{0}<1$, there is no positive equilibrium,and the disease-free equilibrium $P^{0}$ is locally stable.

(2)If $R_{0}>1$, the disease free equilibrium is unstable, the endemic equilibrium $P^{*}$ exists, and it is locally stable.

Proof:The Jacobian matrix at $P^{0}$ is

$$
J\left(P^{0}\right)=\left(\begin{array}{ccccc}
-a_{1} & 0 & -\beta S^{0} & -\beta \alpha S^{0}-\mu \omega \nu & \varepsilon \\
0 & -a_{2} & \beta S^{0} & \beta \alpha S^{0} & 0 \\
0 & \sigma & -a_{3} & 0 & 0 \\
0 & \delta & q \gamma_{1} & -a_{4} & 0 \\
\gamma_{2} & 0 & 0 & 0 & -a_{5}
\end{array}\right)
$$

The characteristic equation at $P^{0}$ is as follow,

$$
\begin{gathered}
\left|\lambda E-J\left(P^{0}\right)\right|=\left[\left(\lambda+a_{1}\right)\left(\lambda+a_{5}\right)+\gamma_{2} \varepsilon\right]|A|, \\
A=\left(\begin{array}{ccc}
\lambda+a_{2} & -\beta S^{0} & -\beta \alpha S^{0} \\
-\sigma & \lambda+a_{3} & 0 \\
-\delta & -q \gamma_{1} & \lambda+a_{4}
\end{array}\right)
\end{gathered}
$$

Let $f(\lambda)=\left(\lambda+a_{1}\right)\left(\lambda+a_{5}\right)+\gamma_{2} \varepsilon=\lambda^{2}+\left(a_{1}+a_{5}\right) \lambda+a_{1} a_{5}+\gamma_{2} \varepsilon$, if $\nabla=\left(a_{1}+a_{5}\right)^{2}-4\left(a_{1} a_{5}+\gamma_{2} \varepsilon\right) \geq 0$, there are two solutions, besides, $a_{1} a_{5}+\gamma_{2} \varepsilon>0, a_{1}+a_{5}>0$, therefore, if there exist solutions, they must be negative.

Let $g(\lambda)=|A|=\lambda^{3}+l_{1} \lambda^{2}+l_{2} \lambda+l_{3}$, where

$$
\begin{gathered}
l_{1}=a_{2}+a_{3}+a_{4}>0, \\
l_{2}=a_{2} a_{3}+a_{2} a_{4}+a_{3} a_{4}-\beta \sigma S^{0}-\beta \alpha \delta S^{0}>0, \\
l_{3}=a_{2} a_{3} a_{4}-a_{4} \beta \sigma S^{0}-a_{3} \beta \alpha \delta S^{0}-\beta \alpha \sigma q \gamma_{1} S^{0}=a_{2} a_{3} a_{4}\left(1-R_{0}\right)>0 \\
\text { When } \mathrm{R}_{0}<1 \text {, we have } \frac{a_{4} \beta \sigma S^{0}+\beta \alpha \sigma q \gamma_{1} S^{0}+a_{3} \beta \alpha \delta S^{0}}{a_{2} a_{3} a_{4}}<1, \\
\frac{a_{3} \beta \alpha \delta S^{0}}{a_{2} a_{3} a_{4}}<1, \frac{a_{4} \beta \sigma S^{0}}{a_{2} a_{3} a_{4}}<1 \\
l_{1} l_{2}-l_{3}=\left(a_{2} a_{3}+a_{2} a_{4}+a_{3} a_{4}\right)\left(a_{2}+a_{3}\right)+\left(a_{2} a_{4}+a_{3} a_{4}\right) a_{4} \\
-\left(a_{2}+a_{3}\right) \beta \sigma S^{0}-\left(a_{2}+a_{4}\right) \beta \alpha \delta S^{0}+\beta \alpha \sigma q \gamma_{1} S^{0} \\
>2 a_{2} a_{3} a_{4}+a_{3}^{2} a_{4}+a_{3} a_{4}^{2}>0 .
\end{gathered}
$$


Therefore, by Routh-Hurwitz criteria, all roots have negative real parts, and $P^{0}$ is locally stable.

Next we will discuss the properties of the endemic equilibrium $P^{*}$, the jacobian matrix at $P^{*}$ is $J\left(P^{*}\right)$

$$
J\left(P^{*}\right)=\left(\begin{array}{ccccc}
-\beta\left(I^{*}+\alpha C^{*}\right)-a_{1} & 0 & -\beta S^{*} & -\beta \alpha S^{*}-\mu \omega \nu & \varepsilon \\
\beta\left(I^{*}+\alpha C^{*}\right) & -a_{2} & \beta S^{*} & \beta \alpha S^{*} & 0 \\
0 & \sigma & -a_{3} & 0 & 0 \\
0 & \delta & q \gamma_{1} & -a_{4} & 0 \\
\gamma_{2} & 0 & 0 & 0 & -a_{5}
\end{array}\right)
$$

We make an elementary row-transformation for $J\left(P^{*}\right)$, and we obtain the following matrix $J^{*}$.

$J^{*}=\left(\begin{array}{ccccc}-\beta\left(I^{*}+\alpha C^{*}\right)-a_{1} & 0 & -\beta S^{*} & -\beta \alpha S^{*}-\mu \omega v & \varepsilon \\ 0 & -a_{2} & M_{1} & \alpha M_{1}-\mu \omega v M_{2} & \varepsilon M_{2} \\ 0 & 0 & -a_{3}+\frac{\sigma}{\sigma_{2}} M_{1} & \frac{\sigma}{\sigma_{2}}\left(\alpha M_{1}-\mu \omega v M_{2}\right) & \frac{\sigma \varepsilon}{\sigma_{2}} M_{2} \\ 0 & 0 & 0 & M_{3}-\mu \omega v M_{4} & \varepsilon M_{4} \\ 0 & 0 & 0 & 0 & M_{5}\end{array}\right)$

Where

$$
\begin{gathered}
M_{1}=\frac{a_{1} \beta S^{*}}{\beta\left(I^{*}+\alpha C^{*}\right)+a_{1}}, M_{2}=\frac{\beta\left(I^{*}+\alpha C^{*}\right)}{\beta\left(I^{*}+\alpha C^{*}\right)+a_{1}} \\
M_{3}=-a_{4}+\alpha M_{1}\left(\delta+\frac{a_{2} q \gamma_{1}+\delta M_{1}}{a_{2} a_{3}-\sigma M_{1}}\right), \\
M_{4}=-\frac{\delta}{a_{2}} M_{2}+\frac{\delta}{a_{2}} \frac{a_{2} q \gamma_{1}+\delta M_{1}}{a_{2} a_{3}-\sigma M_{1}}, \\
M_{5}=-\frac{\gamma_{2}\left(\beta \alpha S^{*}+\mu \omega v\right)}{\beta\left(I^{*}+\alpha C^{*}\right)+a_{1}}-\frac{\gamma_{2} \beta S^{*}}{\beta\left(I^{*}+\alpha C^{*}\right)+a_{1}} \frac{\varepsilon M_{4}}{\mu \omega v M_{4}-M_{3}}
\end{gathered}
$$

The eigenvalues are:

$$
\begin{aligned}
& \lambda_{1}=-\beta\left(I^{*}+\alpha C^{*}\right)-a_{1}<0, \lambda_{2}=-a_{2}<0, \\
& \lambda_{3}=-a_{3}+\frac{\sigma}{a_{2}} M_{1}, \lambda_{4}=M_{3}-\mu \omega v M_{4}, \lambda_{5}=M_{5} .
\end{aligned}
$$

Next we will check $\lambda_{3}, \lambda_{4}, \lambda_{5}<0$. Since $P^{*}$ is the solution of (2.2), then we obtain $a_{2}=\frac{\beta\left(I^{*}+\alpha C^{*}\right) S^{*}}{E^{*}}, a_{3}=\frac{\sigma E^{*}}{I^{*}}$,

$$
\begin{gathered}
\lambda_{3}=-\frac{\sigma E^{*}}{I^{*}}+\frac{\sigma E^{*}}{\beta\left(I^{*}+\alpha C^{*}\right) S^{*}} \frac{a_{1} \beta S^{*}}{\beta\left(I^{*}+\alpha C^{*}\right)+a_{1}} \\
\lambda_{3}<0 \Leftrightarrow \frac{a_{1} I^{*}}{\left[\beta\left(I^{*}+\alpha C^{*}\right)+a_{1}\right]\left(I^{*}+\alpha C^{*}\right)}<1 \Leftrightarrow \beta\left(I^{*}+\alpha C^{*}\right)^{2}+a_{1} \alpha C^{*}>0,
\end{gathered}
$$

it holds as the endemic equilibrium $P^{*}$ exists, at the same time we can know $M_{4}>0$. In addition, $C^{*}$ and $I^{*}$ satisfy

$$
\begin{gathered}
q \gamma_{1}=\frac{a_{4} C^{*}}{I^{*}}-\frac{\delta a_{3}}{\sigma}, a_{4}=\frac{\delta E^{*}+q \gamma_{1} I^{*}}{C^{*}} \\
\lambda_{4}=M_{3}-\mu \omega v M_{4}=-a_{4}+\frac{1}{a_{2}}\left(\alpha M_{1}-\mu \omega v M_{2}\right)\left(\delta+\sigma \frac{a_{2} q \gamma_{1}+\delta M_{1}}{a_{2} a_{3}-\sigma M_{1}}\right)
\end{gathered}
$$

As the abo e, we can obtain that $\lambda_{4}<0$,so $\mu \omega v M_{4}-M_{3}>0$, due to $M_{4}>0$ at the same time, we have $\lambda_{5}=M_{5}<0$. Therefore, all eigenvalues are negative, and we have the $P^{*}$ is locally asymptotically stable.

In order to study the global stability of the disease-free equilibrium, we apply the novel approach in Kamgang and Sallet (2008)[7].

Before proving the main theorem we first give a lemma.

Lemma 4.1 If the following hypothesis $\mathrm{H}_{1}-\mathrm{H}_{5}$ are satisfied, the disease-free equilibrium (DFE) is globally asymptotically stable for system

$$
\begin{aligned}
& \dot{X}_{1}=A_{1}(X)\left(X_{1}-X_{1}^{*}\right)+A_{12}(X) X_{2} \\
& \dot{X}_{2}=A_{2}(X) X_{2}
\end{aligned}
$$

On the positively invariant set $\Omega$, where $X=\left(X_{1}, X_{2}\right)$ and $X^{*}=\left(X_{1}^{*}, 0\right)$ denote a disease-free equilibrium(DFE) of the system above.The variable $X_{1}$ denotes the numbers in the different compartments of susceptible, immune, recovered individuals in other words all the individuals who are not infected and who are not transmitting the disease. The varibal $X_{2}$ denotes the number of infected individuals, For example, exposed individuals, infectious, carrying individuals and so on.

$\mathrm{H}_{1}$ : The system is defined on a positively invariant set $\Omega$ of the nonnegative orthant. The system is dissipative on $\Omega$

$\mathrm{H}_{2}$ : The sub-system $\dot{X}_{1}=A_{1}(X, 0)\left(X-X_{1}^{*}\right)+A_{12}(X) X_{2}$ is globally asymptotically stable at the equilibrium $X_{1}^{*}$ on the canonical projection of $\Omega$.

$\mathrm{H}_{3}$ : The matrix is Metzler and irreducible for any given $X \in \Omega$.

$\mathrm{H}_{4}$ : There exists an upper-bound matrix $\bar{A}_{2}$ for $\mathfrak{R}=\left\{A_{2}(X) / X \in \Omega\right\}$ with the property that $\bar{A}_{2}=\max _{\Omega} \mathfrak{R}$ then for any $\bar{X} \in \Omega$, such that $\dot{X}_{2}=A_{2}(X) X_{2}$ (i. e, the point where the maximum is realized are contained in the diseasefree sub-manifold).

$\mathrm{H}_{5}: \alpha\left(\bar{A}_{2}\right) \leq 0$, where $\alpha\left(\bar{A}_{2}\right) \leq 0$ is spectral bound of $\bar{A}_{2}$.

Using the lemma (4.1), we can obtain that DFE is globally asymptotically stable ,next, we will prove that. 
Proof: Set $X_{1}=(S, V)^{T}, X_{2}=(E, I, C)^{T}, X=\left(X_{1}, X_{2}\right)$ The invariant domain $\Omega$ is obviously positively compact set.

$$
\begin{gathered}
\dot{X}_{1}=A_{1}(X)\left(X_{1}-X_{1}^{*}\right)+A_{12}(X) X_{2} \\
A_{1}(X)=\left(\begin{array}{cc}
-a_{1} & \varepsilon \\
\gamma_{2} & -a_{5}
\end{array}\right), X_{1}^{*}=\left(\begin{array}{c}
\frac{\varepsilon+\mu \omega}{\varepsilon+a_{1}} \\
\frac{a_{1}-\mu \omega}{a_{1}+\varepsilon}
\end{array}\right)
\end{gathered}
$$

This is a linear system which is globally asymptotically stable at $X_{1}^{*}$, the hypothesis $\mathrm{H}_{1}, \mathrm{H}_{2}$ are satisfied.

$$
\begin{gathered}
\dot{X}_{2}=A_{2}(X) X_{2} \\
A_{2}(X)=\left[\begin{array}{ccc}
-a_{2} & \beta S & \beta \alpha S \\
\sigma & -a_{3} & 0 \\
\delta & q \gamma_{1} & -a_{4}
\end{array}\right]
\end{gathered}
$$

As required by hypothesis $\mathrm{H}_{3}$, for any $X \in \Omega$ the matrix $A_{2}(X)$ is Metzler and ireducible.

Now, let we check hypothesis $\mathrm{H}_{4}$. There is a maximum which is uniquely realized in $\Omega$ when $x \in\left(S^{0}, V^{0}\right)$, this corresponds to $P^{0}$, the maximum $A_{2}(\bar{X})$ is given by

$$
A_{2}(\bar{X})=\left[\begin{array}{ccc}
-a_{2} & \beta S^{0} & \beta \alpha S^{0} \\
\sigma & -a_{3} & 0 \\
\delta & q \gamma_{1} & -a_{4}
\end{array}\right]
$$

The hypothesis $\mathrm{H}_{5}$ requires that $\alpha\left(\bar{A}_{2}\right) \leq 0$. Writing $A_{2}(\bar{X})$ as a block matrix

$$
A_{2}(\bar{X})=\left(\begin{array}{ll}
A & B \\
C & D
\end{array}\right)
$$

where

$$
A=-a_{2}, B=\left(\beta S^{0}, \beta \alpha S^{0}\right), C=\left(\begin{array}{l}
\sigma \\
\delta
\end{array}\right), D=\left(\begin{array}{cc}
-a_{3} & 0 \\
q \gamma_{1} & -a_{4}
\end{array}\right)
$$

Since $A$ is already a Metzler stable matrix, the condition $\alpha\left(A_{2}(\bar{X})\right) \leq 0 \quad$ is $\quad$ equal $\quad$ to $\quad \alpha\left(D-C A^{-1} B\right) \leq 0$ $M=D-C A^{-1} B=\left(\begin{array}{cc}-a_{3}+\frac{\sigma}{a_{2}} \beta S^{0} & \frac{\sigma}{a_{2}} \beta \alpha S^{0} \\ q \gamma_{1}+\frac{\delta}{a_{2}} \beta S^{0} & \frac{\delta}{a_{2}} \beta \alpha S^{0}\end{array}\right)$.

The characteristic equation of $\mathrm{M}$ is given by

$$
\begin{gathered}
|\lambda E-M|=\lambda^{2}+d_{1} \lambda+d_{0} \\
d_{1}=\frac{1}{a_{2}}\left(a_{2} a_{3}+a_{2} a_{4}-\beta \sigma S^{0}-\beta \alpha \delta S^{0}\right), \\
d_{0}=\frac{1}{a_{2}}\left(a_{2} a_{3} a_{4}-a_{3} \beta \alpha \delta S^{0}-a_{4} \beta \sigma S^{0}-\beta \alpha \sigma q \gamma_{1} S^{0}\right) .
\end{gathered}
$$

When $R_{0}<1$, we have $d_{1}, d_{0}>0$.It follows from the Routh-Hurwitz criterion that the two eigenvalues have negative real part if and only if $R_{0}<1$. When $\mathrm{R}_{0}=1$, one eigenvalue is zero and another is negative real part root. Hence, $M$ is a stable Metzler matrix if and only if $R_{0} \leq 1$, that is $\alpha\left(A_{2}\right) \leq 0$.We have seen that the hypotheses all are satisfied. Then by Lemma 4.1, we get the theorem 4.2.

Theorem 4.2 For the system (2.2), the disease-free equilibrium $P^{0}$ is globally asymptotically stable if $R_{0}<1$.

\section{CONCLUSIONS}

Hepatitis B is one of the top three infectious diseases reported by the Ministry of Health of China. Almost a third of the people infected with HBV worldwide in China. In this paper, we propose a mathematical model to study the transmission dynamics. We discussed the stability of the disease-free and disease-endemic equilibrium of the model. By analysis, we obtain that vaccine is important to control the hepatitis B virus. At last, we proposed a new method to prove the globally stability of disease-free equilibrium, the method effective for the high dimension ordinary differential equations.

\section{ACKNOWLEDGMENTS}

This work is fully supported by the National Sciences Foundation of China(11301491), the Top Young Academic Leaders of Higher Learning Institutions of Shanxi and the National Sciences Foundation of Shanxi Province (2012011002-1).

\section{REFERENCES}

[1] C. J. Long,,H .Qi and S.H. Huang, "Mathematical modeling of cytotoxic lymphocytemediated Immune response to hepatitis B virus infection," Journal of Biomedicine and Biotechnology,vol.2,pp.160-169,2008.

[2] S. Eikenberry,J.D. Nagy, "The dynamic of a delay model of hepatitis B virus infection with logistic Hepatocyte growth,"Mathematical Biosciences and Engineering, vol.6),pp.283-299, 2009.

[3] J.Q. Li,K.F. Wang, and Y.L. Yang, "Dynamical behaviors of an HBV infection model with logistic hepatocyte growth, "Mathematical and computer modeling,vol.54, pp.704-711,2011.

[4] C.L.Cao, "The difference between acute and chronic hepatitis B ,Liver Doctor,vol.4, pp.17-18, 2010.

[5] J.C.Kamgang and G.Sallet, "Computation of threshold conditions for epidemiological models and global stability of the disease-free equilibrium(DFE), "Mathematical Biosciences, vol.213, pp.1-12,2008.

[6] G.F.Medley,N.A.Lindop,W.J.Edmunds,Heterogeneity,catastrophic dynamics and control,Nat.Med.,7(2001),619-624.

[7] Van den Driessche,P.and Watmough,J., "Reproduction numbers and sub-threshold endemic equilibria for compartmental models of disease transmission",Math. Biosci, vol.80,pp.290-292,2002. 